\title{
Use of unmanned aerial vehicles in crime scene investigations - novel concept of crime scene investigations
}

\section{Introduction}

We live in a world of dynamically changing effects such as human activity, weather patterns and traffic vehicles, and most importantly, in medico-legal point of view, varying types of crimes. Crime scene investigation is a multi-faceted exercise which involves not only gathering trace evidence but also scene recording and documentation, scene reconstruction, etc. In addition to this safety assessment of the scene is vital in some instances. The photographic documentation of crime scenes is the cornerstone of any criminal investigation. ${ }^{1}$ During crime scene investigation, the investigator faces many difficulties due to the complicated nature of the crime scene. In some instances, the particular nature of the crime scene makes it a difficult task for proper examination. For example, the geographical location of the scene like river banks, cliff edges, high rising buildings makes it an extremely difficult exercise. Police and other law enforcement agencies must be able to quickly and effectively secure a crime scene and preserve evidence for use in court and criminal proceedings. In the event of a major crime such as a homicide, getting evidence quickly becomes an extremely critical problem. Footprints, tire tracks and other evidence that is easy to disturb may be left at the crime scene, making walking through the area undesirable. One might think how we could avoid these practical difficulties. Technology of the twenty - first century has an answer to these problems in the form of unmanned aerial vehicles (UAVs) in crime scene investigation. In this paper, the concept of using unmanned aerial vehicles is presented in crime scene investigations. UAVs could be used not only for crime scene investigation but also in exercises like traffic accident investigation, traffic patrol assistance and traffic congestion analysis, explosive ordnance disposal, crowd control applications, disaster response, fire damage assessment, fire scene control, fire Investigation etc. This makes the use of UAV's in police and forensic investigation extremely convenient.

\section{Objectives}

a. Evaluate the feasibility and accuracy of the method concerned.

b. Determine the logistical implementation and quality assurance of the method.

c. Determine the value of the method in crime scene investigation.

d. Determine the need of training staff.

\section{Discussion}

Unmanned aerial vehicle (UAV) is a sophisticated and advanced tool in the field of robotics, aeronautics and electronics. An UAV, technological timeline shows the development over the period of almost three decades. It began in the 1980's as remotely piloted vehicles (RPV) and by the 1990's evolved into UAV's where an operator on ground can take over intermittently as necessary for course correction and later in the decade towards complete automation of the system. The period after 2005 brought forward the era of unmanned aerial systems with sophisticated technology. There are several methods of
Volume 4 Issue I - 2017

\author{
Mendis NDNA,' Dharmarathne TSS, ${ }^{2}$ \\ Wanasinghe $\mathrm{NC}^{\prime}$ \\ 'Department of Forensic Medicine and Toxicology, Sri Lanka \\ 2Department of Orthopedics, Sri Lanka
}

Correspondence: NandithaAselaMendis, Department of Forensic Medicine and Toxicology, Faculty of Medicine, University of Colombo, Sri Lanka, Tel 0094-7I-44I5099 Email ndnamendis@yahoo.co.uk

Received: October 17, 2016 | Published: January 6, 2017

technology that can be used to make measurements. Photogrammetry is one such method which is the science of making measurements from photographs. The output of photogrammetry is typically a map, drawing, measurement, or a 3D model of some real-world object or scene. Contrary to photogrammetry, UAVs have wider use than simple photogrammetry. LIDAR (light illumination detection and ranging) is a technology used in UAVs that uses UV-rays, visible rays and near infra-red light to image objects. Infrared imaging is suitable for a wide range of forensic applications. ${ }^{2}$ It can image wide range of materials including metallic and non-metallic objects, aerosol, clouds and even single molecule. In forensic practice UAVs have many applications like taking photographs of the scene, videotaping, searching for evidences, crime scene reconstruction/mapping the crime scene and investigation. ${ }^{3}$

How can we use UAVs in crime scene investigation? The current procedures involved in crime scene investigation are extensive, time consuming, vulnerable to contamination and need trained personnel. However introducing and applying a novel method for a long established procedure will not be an easy task but UAV is a very powerful tool that can be used in forensic investigations. Therefore we must consider using this technology in the future. Most of the western countries are moving towards this technology as it offers them higher degree of flexibility with added advantages over manual methods of investigations. UAV is a multipurpose tool which can perform various tasks in crime scene investigation. Among them are photography, videotaping, search for evidence, safety assessment, examination of sites which are difficult or near impossible to reach, etc. As an UAV with higher degree of features could transmit photographs to the ground control unit they could be analysed and studied by experts at the lab and the method of scene examination could be determined quite early. This definitely offers an advantage for the crime scene investigators as they could scan the scene before going there in person and also obtaining a second opinion from another expert that would also help in better crime scene management. Use of UAVs can minimize contamination of the scene and also could map and examine the particular scene before visiting it. Therefore, determination of the 
extent of the scene, and determination of entry and exit point to the scene can be decided even before reaching the scene. ${ }^{4}$ As this is not possible under conventional methods of examination it will be very advantageous and helpful in proper scene management.

Are there any other advantages of using UAVs in crime scenes? The answer to this question is yes. Aerial photography is a very helpful method of scene documentation which is useful in scene description and assessment. Until recently there were only a few methods to get an aerial photograph - climbing up the ladder of a fire fighting truck or to a high rise building and by using a manned aircraft, something which in not readily available. UAV is a better solution for this problem as well. As they are smaller in size and readily available (if you have one with you and also purchasing it for an institution is not very costly) you are also able to get better photographs and even close-up photographs. This is advantageous in comparison to the old methods of photography mentioned above. Aerial photographs also give the investigator a "bird's eye view". Aerial photographs provide very good evidentiary material for court. The investigator may be able to articulate his/her evidence in one or two photographs as opposed to 20 or 30 that we took before. It also gives the investigator a different perspective of the scene and is often found to be more advantageous than routine ground level photography. With some advanced types of UAVs, it also helps in identifying recently disturbed ground and even some buried materials. This will be very useful in exercises like mass grave excavations. ${ }^{5}$ Some institutions integrate aerial photographs into a special drawing software programs, which then allows to use the real photograph as the layer on the drawing as opposed to using Google Earth (which is hard because we don't know when the photo was taken).

Is this all what an UAV could do? Definitely no. There are other uses of UAV which are useful for forensic investigation. For instance, a fingerprint found at the crime scene can be imaged and can be sent to the fingerprint bureau directly by the drone. This is very helpful whenever you find it difficult obtain fingerprint sample due to practical reasons. Obtaining real-time, high-quality digital photographs of collision scenes when the vehicles and debris are still at the scene is very useful in assessing the scene very quickly and releasing the scene and shortening the period of road closure avoiding traffic congestions. ${ }^{6}$ It is also used in assessing the reasons for traffic congestions and helps in managing acute crisis situations. UAVs also could be used in emergency response situations like tracing and looking for a missing person. Different other types of disaster responses like fire scene control, disaster response, and explosive disposal could be effectively managed by the use of UAVs. ${ }^{7}$ The UAV is a resourceful tool in crime scene investigation and management. We also should consider moving towards this technology since it holds the future of crime scene investigation. Next, it is important to determine is the feasibility of the method concern. Financially, a basic type of UAV is not very expensive. A basic model would cost around $\$ 1000-2000$. However, a more sophisticated unit would probably cost around $\$ 3000-4000$. This means that it is not big financial burden on an institution carrying out crime scene investigation and other medico legally important work. We don't have a much problems about the accuracy of certain methods carried out by the UAV because technical details and capabilities of a particular model or unit could be ascertained before purchasing it. Quality assurance is an important aspect that we should consider. Without maintaining proper standards both of the equipment and the methods employed the legal validity of the outcome could be questioned. However if proper standardized equipment is purchased maintaining quality will not be a big issue to be concerned with. The next issue is to decide actually whether this proposed method offers valuable contribution towards improving the existing methods. As we don't have any firsthand information about the method, we have to base our decision on information available from institutions who use them. However, available documents and information indicate that the technique to be very valuable.

\section{Conclusion}

UAV could serve as a bridge between the investigators in the lab and the crime scene. The laboratory can receive all the data, live videos, and images of the crime scene on real time basis and can further direct the officer to collect specific evidence or relevant data. Thus, use of UAVs could greatly enhance the efficacy of crime scene investigation. It also helps to gain access to inaccessible area and also to retrieve evidence/samples from such areas. As mentioned earlier obtaining photographs of fingerprints could be used very effectively in tracing the assailant in some cases. The feasibility of the method should be assessed on an institutional basis and decision made with regard to using this technique.

\section{Acknowledgments}

None.

\section{Conflicts of interest}

None.

\section{References}

1. Houck MM. Review of: Advanced Crime Scene Photography. J Forensic Sci. 2010;55(6):1675.

2. Edelman GJ, Hoveling RJ, Roos M, et al. Infrared Imaging of the Crime Scene: Possibilities and Pitfalls. J Forensic Sci. 2013;58(5):1156-1162.

3. Mishra V, Dedhia H, Wavhal S. Application of Drones in the Investigation and Management of a Crime Scene. Forensic Science. 2015;4(4):1-2.

4. Drones add new dimension to crime scene investigation. The Huffington Post, USA, 2014.

5. Gashi B, Edwards MR, Sermon PA, et al. Measurement of $9 \mathrm{~mm}$ cartridge case external temperatures and its forensic application. Forensic Sci Int. 2010;200(1-3):21-27.

6. Police crime investigations now use drones. Drone Makers, UK.

7. Almog J. Forensic science does not start in the lab: the concept of diagnostic field tests. J Forensic Sci. 2006;51(6):1228-1234. 